\title{
The density of the time to ruin for a Sparre Andersen process with Erlang arrivals and exponential claims
}

\author{
David C M Dickson, Barry D Hughes and Zhang Lianzeng
}

\begin{abstract}
We derive expressions for the density of the time to ruin given that ruin occurs in a Sparre Andersen model in which individual claim amounts are exponentially distributed and inter-arrival times are distributed as $\operatorname{Erlang}(n, \beta)$. We provide numerical illustrations of finite time ruin probabilities, as well as illustrating features of the density functions.
\end{abstract}

\section{Introduction}

The Sparre Andersen surplus process $\{U(t)\}_{t \geq 0}$ is defined by

$$
U(t)=u+c t-\sum_{i=1}^{N(t)} X_{i}
$$

where $u$ is the initial surplus, $c$ is the rate of premium income per unit time, $\left\{X_{i}\right\}_{i=1}^{\infty}$ is a sequence of independent and identically distributed (i.i.d.) random variables, where $X_{i}$ represents the amount of the $i$ th claim, and $\{N(t)\}_{t \geq 0}$ is a counting process with $N(t)$ denoting the number of claims up to time $t$. The sequence of i.i.d. random variables $\left\{W_{i}\right\}_{i=1}^{\infty}$ represents the claim interarrival times, with $W_{1}$ being the time until the first claim, and we assume that claim amounts are independent of claim inter-arrival times. Further, we assume that $c E\left(W_{1}\right)>E\left(X_{1}\right)$, and define the premium loading factor $\varpi$ by $c E\left(W_{1}\right)=(1+\varpi) E\left(X_{1}\right)$.

We denote by $p$ the density function of $X_{1}$, and throughout this paper we let $p(x)=\alpha \exp \{-\alpha x\}, x>0$. We also assume that $W_{1}$ has an $\operatorname{Erlang}(n, \beta)$ distribution, so that

$$
\operatorname{Pr}\left(W_{1} \leq t\right)=1-e^{-\beta t} \sum_{j=0}^{n-1} \frac{(\beta t)^{j}}{j !}
$$

with density function $\beta^{n} t^{n-1} e^{-\beta t} /(n-1)$ !, and hence the premium loading factor becomes $\varpi=c \alpha n / \beta-1$.

Let $T$ denote the time of ruin, and be given by

$$
T=\left\{\begin{array}{l}
\inf \{t: U(t)<0\} \\
\infty \text { if } U(t) \geq 0 \text { for all } t>0
\end{array}\right.
$$


and let $T_{c}=T \mid T<\infty$. The probability of ultimate ruin from initial surplus $u$ is defined as

$$
\psi(u)=\operatorname{Pr}[T<\infty \mid U(0)=u] .
$$

Similarly, the probability of ruin by time $t, t>0$, is defined as

$$
\psi(u, t)=\operatorname{Pr}[T \leq t \mid U(0)=u] .
$$

We define the function $\phi$ by

$$
\phi(u)=E\left[e^{-\delta T} I(T<\infty) \mid U(0)=u\right]
$$

where $\delta$ is a non-negative parameter and $I$ is the indicator function. From Gerber and Shiu (1998) we have

$$
\phi(u)=\int_{0}^{\infty} e^{-\delta t} \frac{\partial}{\partial t} \psi(u, t) d t .
$$

In the case of the classical risk model, i.e. when $W_{1}$ has an exponential distribution, it is well known that an explicit solution for $\psi(u, t)$ exists when $X_{1}$ has an exponential distribution. See Seal (1978) and references therein. More recently, attention has focussed on finding the density of $T_{c}$ by using $\phi$ as a Laplace transform and inverting that transform. Garcia (2002) uses the complex inversion formula to produce explicit solutions for $\psi(u, t)$ when $X_{1}$ has an exponential distribution and when it has an Erlang(2) distribution. Drekic and Willmot (2003) derive the density of $T_{c}$ in the case when $X_{1}$ has an exponential distribution by direct inversion of $\phi$.

We start with some preliminary results in the next section. Then, in Section 3 , we apply the ideas contained in Garcia (2002) to derive the density of $T_{c}$ when $X_{1}$ has an exponential distribution and $W_{1}$ is distributed as $\operatorname{Erlang}(n, \beta)$. In particular, we derive a more readily computable form of the density of $T_{c}$ than the Drekic-Willmot solution when $n=1$. Garcia's basic approach, while producing correct answers, involves unjustified steps which are here made completely rigorous. Finally, the results of Section 3 are illustrated in Section 4.

\section{Preliminaries}

We start by establishing Lundberg's fundamental equation for our model. The arguments that follow are essentially those of Gerber and Shiu (1998), who consider the classical risk model, but modified to our circumstances. Define

$$
V(m)=u+\sum_{i=1}^{m}\left(c W_{i}-X_{i}\right)
$$

for $m=1,2,3, \ldots$ with $V(0)=u$, so that $V(m)$ gives the surplus at the time of the $m$ th claim, and let

$$
\tau(m)=\sum_{i=1}^{m} W_{i}
$$

denote the time of the $m$ th claim, with $\tau(0)=0$. Then by standard arguments the process

$$
\{\exp \{-\delta \tau(m)+\xi V(m)\}\}_{m=0}^{\infty}
$$


is a martingale if

$$
E\left[\exp \left\{-(\delta-c \xi) W_{1}\right\}\right] E\left[\exp \left\{-\xi X_{1}\right\}\right]=1,
$$

which becomes

$$
\left(\frac{\beta}{\beta+\delta-c \xi}\right)^{n} \frac{\alpha}{\alpha+\xi}=1
$$

for our choice of distributions for $W_{1}$ and $X_{1}$. Further, there is a unique negative value $\xi=-R$ where $R>0$ which satisfies equation (2.1), which is Lundberg's fundamental equation for our model.

By applying the optional stopping theorem we find that

$$
\phi(u)=\left(1-\frac{R}{\alpha}\right) e^{-R u}
$$

a result which is derived by this argument by Gerber and Shiu (1998) when the Erlang parameter $n$ is 1, and by a different argument by Dickson and Hipp (2001) when the Erlang parameter $n$ is 2 .

When $\delta=0$, we get the well known result

$$
\psi(u)=\left(1-\frac{R_{0}}{\alpha}\right) e^{-R_{0} u}
$$

where $R_{0}$ is the adjustment coefficient. See, for example, Grandell (1991).

\section{Main Results}

Applying results from the previous two sections to our model we have

$$
\int_{0}^{\infty} e^{-\delta t} \frac{\partial}{\partial t} \psi(u, t) d t=\left(1-\frac{R}{\alpha}\right) e^{-R u}
$$

where

$$
\left(\frac{\beta}{\beta+\delta+c R}\right)^{n} \frac{\alpha}{\alpha-R}=1 .
$$

As $R$ is a function of $\delta$, the right hand side of equation (3.1) is a function of $\delta$ and hence we can find $(\partial / \partial t) \psi(u, t)$ if we can invert the Laplace transform.

Drekic and Willmot (2002) have found the inverse Laplace transform for the case $n=1$ by first expanding the right-hand side of equation (3.1) in a series in powers of $1-R / \alpha$ and then inverting the Laplace transform term-by-term. Briefly, if one notes that for $n=1$ the unique solution of equation (3.2) with $0<R<\alpha$ corresponds to

$$
1-\frac{R}{\alpha}=\frac{\beta+\delta+\alpha c-\sqrt{(\beta+\delta+\alpha c)^{2}-4 \alpha \beta c}}{2 \alpha c},
$$

each term in the expansion can be inverted in terms of the modified Bessel function $I_{\nu}$ (Abramowitz and Stegun 1965, Chapter 9) and one finds from this that $^{1}$

$$
\frac{\partial}{\partial t} \psi(u, t)=\frac{e^{-\alpha u-(\alpha c+\beta) t}}{\alpha u t} \sum_{n=0}^{\infty} \frac{(n+1)}{n !}\left[u(\alpha \beta / c)^{1 / 2}\right]^{n+1} I_{n+1}\left((4 \alpha \beta c)^{1 / 2} t\right) .
$$

\footnotetext{
${ }^{1}$ To convert to the notation of Drekic and Willmot, set $\alpha u=\mu x /(1+\theta), \beta=\lambda(1+\theta)$ and
} $\alpha c=\lambda$. 
This result, though readily computable, is not especially convenient and the technique does not generalise to $n>1$, where the solution for $1-R / \alpha$ is either too unpleasant to permit term-by-term inversion $(n=2,3,4)$ or unable to be found analytically $(n \geq 5)$.

Garcia (2002) has noted that the Laplace transform encountered in the present context may also be evaluated using the complex inversion formula for $n=1$, and we shall follow this approach here for arbitrary positive integer $n$. As certain subtleties are encountered when using complex variable methods, we shall first briefly summarise the important relevant aspects of the complex variable theory of the Laplace transform (Sneddon 1972, Chapter 3; Henrici 1977, Chapter 10).

Let $f(t)$ be a function defined for $t>0$ with at most finitely many points of discontinuity. If the Laplace transform integral

$$
\int_{0}^{\infty} e^{-s t} f(t) d t=\widehat{f}(s)
$$

exists for $s=s_{0}$, then the integral exists also at least for all complex $s$ with $\operatorname{Re}\{s\}>\operatorname{Re}\left\{s_{0}\right\}$, and the resulting function $\widehat{f}(s)$ is holomorphic in the halfplane in which $\operatorname{Re}\{s\}>\operatorname{Re}\left\{s_{0}\right\}$. For any fixed real number $\kappa$ with $\kappa>\operatorname{Re}\left\{s_{0}\right\}$, the function $f(t)$ can be recovered from $\widehat{f}(s)$ by a contour integral taken along the 'Bromwich contour', which is the line $\operatorname{Re}\{s\}=\kappa$, traversed in the direction of increasing $\operatorname{Im}\{s\}$ :

$$
f(t)=\frac{1}{2 \pi i} \int_{\kappa-i \infty}^{\kappa+i \infty} e^{s t} \widehat{f}(s) d s \quad(t>0) .
$$

There are mild regularity conditions on $f(t)$ for the inversion formula to hold as stated, and at points of discontinuity of $f$, the contour integral returns $\left[\lim _{\tau \downarrow t} f(\tau)+\lim _{\tau \uparrow t} f(\tau)\right] / 2$ rather than $f(t)$. Also, if the Bromwich contour integral fails to converge in the classical sense, it is to be interpreted as the limit as $L \rightarrow \infty$ of the contour integral from $\kappa-i L$ to $\kappa+i L$.

In most cases where Laplace transforms are used, the Laplace transform $\widehat{f}(s)$ has an analytic continuation to the rest of the complex plane, apart from certain singular points (singularities), and subject to modest requirements on the decay of $|\widehat{f}(s)|$ as $|s| \rightarrow \infty$, one may complete the integration contour and then usually either

(a) evaluate the integral using the residue theorem, giving

$$
f(t)=\sum_{\text {singularities }} \operatorname{Residue}\left\{e^{s t} \widehat{f}(s)\right\},
$$

this is the case that applies if all singularities of the analytic continuation of $\widehat{f}(s)$ are isolated (that is, poles or isolated essential singularities); or

(b) convert the contour integral to a more tractable equivalent real integral this is the case when the analytic continuation of $\widehat{f}(s)$ has one or two branch points.

We shall scale the problem by writing

$$
\frac{\partial}{\partial t} \psi(u, t)=\beta f(\beta t), \quad s=\frac{\delta}{\beta}, \quad T=\beta t \quad \text { and } \quad \epsilon=\frac{c \alpha}{\beta} .
$$


We shall also set

$$
1-R / \alpha=z^{n},
$$

where from the context of the original problem we have $0<R<\alpha$, so that we have $z$ real, with $z \in(0,1)$ when $s$ is a positive real number. We now have

$$
\widehat{f}(s)=z^{n} \exp \left(-\alpha u+\alpha u z^{n}\right),
$$

where $s$ is to be determined from

$$
z\left[1+s+\epsilon\left(1-z^{n}\right)\right]=1 .
$$

We remark that if we define

$$
P_{s}(z)=\epsilon z^{n+1}-(1+s+\epsilon) z+1,
$$

the relation between $s$ and $z$ is equivalent to $P_{s}(z)=0$. For real, positive $s$ and real $z$, we may observe that $P_{s}(z)$ is real, $P_{s}(0)=1, P_{s}(1)=-s<0$, and $P_{s}(z) \rightarrow \infty$ as $z \rightarrow \infty$, so that $P_{s}(z)=0$ has real solutions in each of the intervals $(0,1)$ and $(1, \infty)$. Moreover, $P_{s}^{\prime}(0)<0$ and $P_{s}^{\prime \prime}(z)>0$ for $z>0$. This ensures that for positive real $s$, equation (3.3) has precisely one real solution in the interval $(0,1)$, and precisely one real solution in the interval $(1, \infty)$. All other solutions are either complex, or are real negative numbers. The Mapping Theorem discussed by Marsden and Hoffman (1999, pp. 399-400) ensures that for each real $s_{0} \in(0, \infty)$, there exists $\Delta\left(s_{0}\right)>0$ such that there is a unique solution $z=Z(s)$ of $P_{s}(z)=0$ in $\left|s-s_{0}\right|<\Delta\left(s_{0}\right)$. The initial analytic continuation of $\widehat{f}(s)$ beyond the positive real axis is therefore unambiguously defined using the root $z=Z(s)$ of equation (3.3) that converges as $s \rightarrow s_{0}$ to the real root $z=Z\left(s_{0}\right) \in(0,1)$ of $P_{s_{0}}(z)=0$.

\section{The case $n=1: s$-plane analysis}

Consider as an illustration the case $n=1$, for which we have as the solutions for real positive $s$ of $P_{s}(z)=0$ the choices

$$
z=\frac{1+s+\epsilon \pm \sqrt{(1+s+\epsilon)^{2}-4 \epsilon}}{2 \epsilon} .
$$

It is easily shown that the negative sign is to be selected to obtain the root in $(0,1)$, and so we have

$$
Z(s)=\frac{1}{2 \epsilon}\left\{1+s+\epsilon-\left(1+s+\epsilon-2 \epsilon^{1 / 2}\right)^{1 / 2}\left(1+s+\epsilon+2 \epsilon^{1 / 2}\right)^{1 / 2}\right\} .
$$

To obtain a valid analytic continuation of $Z(s)$ we assign each of the square roots their principal values. Then if $s=\sigma+i \tau$ we have

$$
\left(1+s+\epsilon \pm 2 \epsilon^{1 / 2}\right)^{1 / 2}=\left[\left(1+\sigma+\epsilon \pm 2 \epsilon^{1 / 2}\right)^{2}+\tau^{2}\right]^{1 / 4} \exp \left(\frac{i \theta_{ \pm}}{2}\right),
$$

where $\theta_{ \pm} \in(-\pi, \pi]$ with

$$
\begin{aligned}
\sin \theta_{ \pm} & =\frac{\tau}{\left[\left(1+\sigma+\epsilon \pm 2 \epsilon^{1 / 2}\right)^{2}+\tau^{2}\right]^{1 / 2}} \\
\cos \theta_{ \pm} & =\frac{1+\sigma+\epsilon \pm 2 \epsilon^{1 / 2}}{\left[\left(1+\sigma+\epsilon \pm 2 \epsilon^{1 / 2}\right)^{2}+\tau^{2}\right]^{1 / 2}}
\end{aligned}
$$



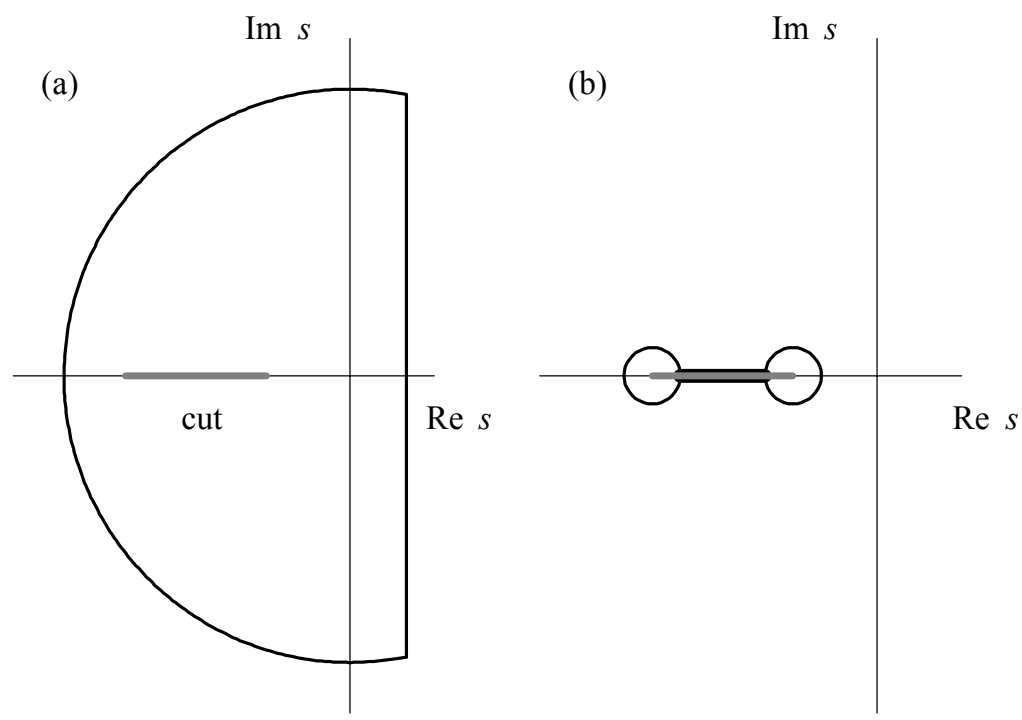

Figure 3.1: Completion and contraction in the $s$-plane of the Bromwich contour $\operatorname{Re}\{s\}=\kappa$. (a) The finite Bromwich contour with $-L \leq \operatorname{Im}\{s\} \leq L$ is completed with a circular arc that encloses completely the branch cut. (b) The resulting closed contour is contracted to circles of radius $\eta$ centred on the branch points, and straight lines along the top and bottom edges of the cut. The contributions from the three circular arcs vanish in the limits $\eta \rightarrow 0$ and $L \rightarrow \infty$.

We need to evaluate

$$
f(T)=\frac{1}{2 \pi i} \int_{\kappa-i \infty}^{\kappa+i \infty} e^{s T} Z(s) \exp (-\alpha u+\alpha u Z(s)) d s \quad(T>0) .
$$

The integrand has branch point singularities at $s=-a$ and $s=-b$, where

$$
a=1+\epsilon-2 \epsilon^{1 / 2}=\left(1-\epsilon^{1 / 2}\right)^{2}, \quad b=1+\epsilon+2 \epsilon^{1 / 2}=\left(1+\epsilon^{1 / 2}\right)^{2} .
$$

If we now consider the limiting behaviour of $Z(\sigma+i \tau)$ as $\tau \rightarrow 0$ we find that $Z(\sigma+i \tau)$ is continuous across $\tau=0$ except for $-b \leq \sigma \leq-a$. We therefore cut the $s$-plane along the straight line joining $a$ and $b$, and note that for $-b<\sigma<-a$

$$
Z(\sigma+i \tau) \rightarrow Z^{ \pm}(\sigma)=\frac{1}{2 \epsilon}\left\{1+\epsilon+\sigma \mp i|b+\sigma|^{1 / 2}|a+\sigma|^{1 / 2} \mid\right\} \quad \text { as } \tau \rightarrow 0^{ \pm}
$$

We complete the contour in the standard manner with a circular arc in the left half-plane and contract the contour down to a 'dumb-bell' shaped contour consisting of the upper and lower surfaces of the branch cut between $s=-b+\eta$ and $s=-a-\eta$ and circles of radius $\eta$ centred on $s=-a$ and $s=-b$ (see Figure 3.1 ). In the limit $\eta \rightarrow 0$, the contributions from the circular arcs vanish (the integrand is bounded, and the contour length vanishes in the limit). Making careful use of equation (3.5) and writing $x=-\sigma$, we find that

$$
f(T)=\frac{1}{2 \pi i} \int_{a}^{b} e^{-x T-\alpha u}\left\{Z^{-}(-x) e^{\alpha u Z^{-}(-x)}-Z^{+}(-x) e^{\alpha u Z^{+}(-x)}\right\} d x .
$$


If we make the change of variable $x=a+(b-a) \xi=1+\epsilon-2 \epsilon^{1 / 2}+4 \epsilon^{1 / 2} \xi$, we find that

$$
Z^{ \pm}(-x)=\epsilon^{-1 / 2}\left\{1-2 \xi \mp 2 i[\xi(1-\xi)]^{1 / 2}\right\},
$$

giving

$$
\begin{aligned}
f(T)=\frac{4 e^{\alpha u\left(\epsilon^{-1 / 2}-1\right)-\left(1+\epsilon-2 \epsilon^{1 / 2}\right) T}}{\pi} \int_{0}^{1} e^{-4 \epsilon^{1 / 2} T \xi-2 \alpha u \epsilon^{-1 / 2} \xi} & {\left[(1-2 \xi) \sin \left\{\frac{2 \alpha u}{\epsilon^{1 / 2}}[\xi(1-\xi)]^{1 / 2}\right\}\right.} \\
& \left.+2[\xi(1-\xi)]^{1 / 2} \cos \left\{\frac{2 \alpha u}{\epsilon^{1 / 2}}[\xi(1-\xi)]^{1 / 2}\right\}\right] d \xi .
\end{aligned}
$$

Re-expressing this result in the original variables, we find that

$$
\begin{aligned}
\frac{\partial}{\partial t} \psi(u, t)=\frac{4 \beta e^{u(\alpha \beta / c)^{1 / 2}-\alpha u-\left[\beta+\alpha c-2(\alpha \beta c)^{1 / 2}\right] t}}{\pi} \int_{0}^{1} e^{-4(\alpha \beta c)^{1 / 2}[t+u /(2 c)] \xi} \\
{\left[(1-2 \xi) \sin \left\{\frac{2 u(\alpha \beta)^{1 / 2}}{c^{1 / 2}}[\xi(1-\xi)]^{1 / 2}\right\}\right.} \\
\left.+2[\xi(1-\xi)]^{1 / 2} \cos \left\{\frac{2 u(\alpha \beta)^{1 / 2}}{c^{1 / 2}}[\xi(1-\xi)]^{1 / 2}\right\}\right] d \xi
\end{aligned}
$$

We can use Watson's Lemma (Olver 1974, Chapter 3; Henrici 1977, Chapter 11) to deduce the asymptotic expansion for this integral as $4(\alpha \beta c)^{1 / 2}[t+u /(2 c)] \rightarrow$ $\infty$. Watson's Lemma states that if $\phi(\xi) \sim \sum_{n=0}^{\infty} \phi_{n} \xi^{\lambda_{n}-1}$ as $\xi \rightarrow 0$ (with $\left.0<\lambda_{0}<\lambda_{1}<\lambda_{2}<\cdots\right)$, then as $Z \rightarrow \infty$,

$$
\int_{0}^{\infty} e^{-Z \xi} \phi(\xi) d \xi \sim \sum_{m=0}^{\infty} \frac{\Gamma\left(\lambda_{m}\right) \phi_{m}}{Z^{\lambda_{m}}},
$$

where $\Gamma(\lambda)=\int_{0}^{\infty} e^{-\xi} \xi^{\lambda-1} d \xi$ is the usual gamma function. The expansion is in general a divergent asymptotic expansion, rather than a convergent series.

From the first term of the Watson's Lemma expansion, on recalling that $\Gamma(3 / 2)=\pi^{1 / 2} / 2$ we deduce that if $4(\alpha \beta c)^{1 / 2}[t+u /(2 c)] \gg 1$,

$$
\frac{\partial}{\partial t} \psi(u, t) \approx \frac{4 \beta\left[1+u(\alpha \beta / c)^{1 / 2}\right] e^{u(\alpha \beta / c)^{1 / 2}-\alpha u-\left[\beta+\alpha c-2(\alpha \beta c)^{1 / 2}\right] t}}{\pi^{1 / 2}\left\{4(\alpha \beta c)^{1 / 2}[t+u /(2 c)]\right\}^{3 / 2}} .
$$

This result has the status of a long-time limit, but for a given large $t$, the difference between the approximation and the true value can be sensitive to the values of parameters $\alpha, \beta, c$ and $u$. Further terms in the expansion are easily derived, but for parameter regimes where the first term alone is inadequate, an alternative expression discussed below is readily computable to very high precision. We remark that the change of variable $\xi=\sin ^{2} \theta(0 \leq \theta \leq \pi / 2)$ enables the solution (3.6) to be rewritten as

$$
\begin{gathered}
\frac{\partial}{\partial t} \psi(u, t)=\frac{4 \beta e^{u(\alpha \beta / c)^{1 / 2}-\alpha u-\left[\beta+\alpha c-2(\alpha \beta c)^{1 / 2}\right] t}}{\pi} \int_{0}^{\pi / 2} e^{-4(\alpha \beta c)^{1 / 2}[t+u /(2 c)] \sin ^{2} \theta} \\
\times \sin \left\{\frac{u(\alpha \beta)^{1 / 2}}{c^{1 / 2}} 2 \sin \theta \cos \theta+2 \theta\right\} 2 \sin \theta \cos \theta d \theta .
\end{gathered}
$$


As the integrand is even, the integration interval can be extended to $(-\pi, \pi)$ and a subsequent change of variable $\phi=2 \theta$ gives

$$
\begin{aligned}
\frac{\partial}{\partial t} \psi(u, t)=\frac{\beta e^{-\alpha u-(\beta+\alpha c) t}}{\pi} \int_{-\pi}^{\pi} & e^{2(\alpha \beta c)^{1 / 2}[t+u /(2 c)] \cos \phi} \\
& \times \sin \left\{\frac{u(\alpha \beta)^{1 / 2}}{c^{1 / 2}} \sin \phi+\phi\right\} \sin \phi d \phi .
\end{aligned}
$$

\section{The case $n=1: z$-plane analysis}

Garcia (2002) avoids the issue of determining $Z(s)$ by making a change of variable in the contour integral, producing an integral over a new complex variable $z$. In our notation the change of variable is

$$
s=z^{-1}+\epsilon z-1-\epsilon .
$$

However, as the change of variable also involves a change of integration contour, and effectively runs the contour through an isolated essential singularity, the argument requires more care. As before, we write $s=\sigma+i \tau$. On the Bromwich contour, we have $\operatorname{Re}\{s\}=\sigma=\kappa$. Observe that as $|\tau| \rightarrow \infty$ for fixed $\sigma=\kappa$ we have

$$
\sin \theta_{ \pm} \rightarrow \operatorname{sgn}(\tau), \quad \cos \theta_{ \pm}=O\left(\tau^{-1}\right),
$$

so that $\theta_{ \pm} \rightarrow \operatorname{sgn}(\tau) \pi / 2$. In more detail,

$$
\sin \theta_{ \pm}=\operatorname{sgn}(\tau)\left[1-\frac{\left(1+\kappa+\epsilon \pm 2 \epsilon^{1 / 2}\right)^{2}}{2 \tau^{2}}+O\left(\tau^{-4}\right)\right],
$$

and so on setting $\theta=\operatorname{sgn}(\tau)[\pi / 2-\eta]$ and examining the small- $\eta$ behaviour, we deduce that

$$
\theta_{ \pm}=\operatorname{sgn}(\tau)\left[\frac{\pi}{2}-\frac{1+\kappa+\epsilon \pm \epsilon^{1 / 2}}{|\tau|}+O\left(\tau^{-2}\right)\right]
$$

giving

$$
\frac{1}{2}\left(\theta_{-}+\theta_{+}\right)=\operatorname{sgn}(\tau)\left[\frac{\pi}{2}-\frac{1+\kappa+\epsilon}{|\tau|}+O\left(\tau^{-2}\right)\right] .
$$

Also,

$$
\begin{aligned}
{\left[\left(1+\kappa+\epsilon \pm 2 \epsilon^{1 / 2}\right)^{2}+\tau^{2}\right]^{1 / 4} } & =|\tau|^{1 / 2}\left[1+\left(1+\kappa+\epsilon \pm 2 \epsilon^{1 / 2}\right)^{2} / \tau^{2}\right]^{1 / 4} \\
& =|\tau|^{1 / 2}\left[1+O\left(\tau^{-2}\right)\right]
\end{aligned}
$$

and it follows, after a little algebra in which many cancellations occur in equation (3.4), that

$$
Z(\kappa+i \tau)=-\frac{i}{\tau}+\frac{1+\kappa+\epsilon}{\tau^{2}}+O\left(\tau^{-3}\right) \quad \text { as } \tau \rightarrow \infty .
$$

The image in the $z$-plane of the Bromwich contour is illustrated in Figure 3.2. Interpreted as a closed contour, it is traversed clockwise and passes through the point $z=0$. However $z=0$ is an isolated essential singularity of

$$
e^{\delta t}=e^{s T}=e^{\left(z^{-1}+\epsilon z-1-\epsilon\right) T} .
$$




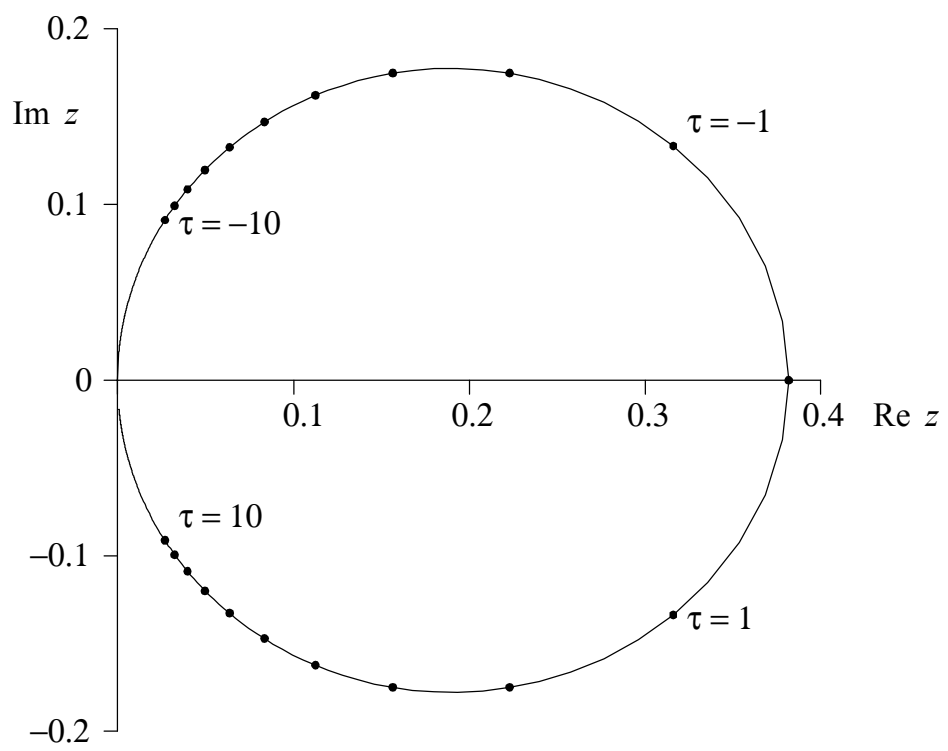

Figure 3.2: The image in the $z$-plane of the Bromwich contour $\operatorname{Re}\{s\}=\kappa$, for the case $n=1, \kappa=1, \epsilon=1$. The discs represent points corresponding to integer values of $\tau=\operatorname{Im}\{s\}$ with $-10 \leq \tau \leq 10$.

Garcia (2002) has not addressed this issue, and has in effect evaluated the contour integral in the $z$-plane by assuming that the contour encircles $z=0$ rather than passing through it. Fortunately, Garcia's analysis can be set on a firm foundation by the following construction.

Let $L_{0}>0$ be given and consider $L \geq L_{0}$ (where ultimately we shall let $L \rightarrow \infty)$. Then the image of the segment $-L \leq \operatorname{Im}\{s\} \leq L$ of the Bromwich contour $\operatorname{Re}\{s\}=\kappa$ in the $s$-plane corresponds in the $z$-plane to a contour with $\operatorname{Re}\{z\} \geq \chi(L)$, where

$$
\begin{aligned}
Z(\kappa \pm i L) & =\chi(L) \mp i \lambda(L), \\
\chi(L) & =\frac{1+\kappa+\epsilon}{L^{2}}+O\left(L^{-3}\right), \\
\lambda(L) & =\frac{1}{L}+O\left(L^{-3}\right) .
\end{aligned}
$$

We consider

$$
\mathcal{I}_{L}=\frac{1}{2 \pi i} \int_{C} e^{\left(z^{-1}+\epsilon z-1-\epsilon\right) T} z e^{-\alpha u+\alpha u z}\left[-\frac{1}{z^{2}}+\epsilon\right] d z,
$$

where the contour $C$, traversed clockwise, consists of four smooth arcs (see Figure 3.3):

$C_{1}$ : the image in the $z$-plane of the segment $-L \leq \operatorname{Im}\{s\} \leq L$ of the Bromwich contour $\operatorname{Re}\{s\}=\kappa$; 
$C_{2}$ : the straight line joining $z=\chi(L)+i \lambda(L)$ to $z=i \lambda(L)$;

$C_{3}$ : the semicircular arc to the left of the origin joining $z=i \lambda(L)$ to $z=$ $-i \lambda(L)$

$C_{4}$ : the straight line joining $z=-i \lambda(L)$ to $z=\chi(L)-i \lambda(L)$.

From the residue theorem, remembering that the contour is traversed clockwise, we have

$$
\begin{aligned}
\mathcal{I}_{L} & =\underset{z=0}{- \text { Residue }}\left\{e^{\left(z^{-1}+\epsilon z-1-\epsilon\right) T} z e^{-\alpha u+\alpha u z}\left[-\frac{1}{z^{2}}+\epsilon\right]\right\} \\
= & e^{-\alpha u-(1+\epsilon) T} \text { Residue }\left\{e^{z^{-1} T} e^{(\epsilon T+\alpha u) z}\left[\frac{1}{z}-\epsilon z\right]\right\} \\
= & e^{-\alpha u-(1+\epsilon) T}\left\{\sum_{r=0}^{\infty} \frac{T^{r}(\epsilon T+\alpha u)^{r}}{(r !)^{2}}-\epsilon \sum_{r=0}^{\infty} \frac{T^{r+2}(\epsilon T+\alpha u)^{r}}{r !(r+2) !}\right\} \\
= & e^{-\alpha u-(1+\epsilon) T}\left\{{ }_{0} F_{1}(1 ; T(\epsilon T+\alpha u))-\frac{\epsilon T^{2}}{2}{ }_{0} F_{1}(3 ; T(\epsilon T+\alpha u))\right\},
\end{aligned}
$$

where

$$
{ }_{0} F_{1}(C ; Z)=\sum_{m=0}^{\infty} \frac{\Gamma(C) Z^{m}}{\Gamma(C+m) m !}
$$

is a special case of the hypergeometric series. Note that these series can also be recognised as modified Bessel functions, since

$$
I_{\nu}(Z)=\left(\frac{Z}{2}\right)^{\nu} \sum_{m=0}^{\infty} \frac{\left(Z^{2} / 4\right)^{m}}{m ! \Gamma(m+\nu+1)}=\frac{1}{\Gamma(\nu+1)}\left(\frac{Z}{2}\right)^{\nu}{ }_{0} F_{1}\left(\nu+1 ; Z^{2} / 4\right)
$$

and so

$$
\mathcal{I}_{L}=e^{-\alpha u-(1+\epsilon) T}\left\{I_{0}(\sqrt{4 T(\epsilon T+\alpha u)})-\frac{\epsilon T}{\epsilon T+\alpha u} I_{2}(\sqrt{4 T(\epsilon T+\alpha u)})\right\} .
$$

We now prove that the contributions from the contour integrals along the arcs $C_{2}, C_{3}$ and $C_{4}$ all vanish in the limit $L \rightarrow \infty$.

On the semicircular $\operatorname{arc} C_{3}$, we may write $z=\lambda(L) e^{i \phi}$ with $\pi / 2 \leq \phi \leq 3 \pi / 2$. Then

$$
\left|e^{\left(z^{-1}+\epsilon z-1-\epsilon\right) T}\right|=e^{\left[\lambda(L)^{-1} \cos \phi+\epsilon \lambda(L) \cos \phi-1-\epsilon\right] T} .
$$

All terms in the argument of the exponential on the right are non-positive and except near the imaginary axis, the exponential vanishes rapidly as $L \rightarrow \infty$. With the constant $K$ depending on $\alpha, u, \epsilon, \kappa, T$ and $L_{0}$, but independent of $L$, we have

$$
\left|\int_{C_{3}} e^{\left(z^{-1}+\epsilon z-1-\epsilon\right) T} z e^{-\alpha u+\epsilon \alpha u z}\left[-\frac{1}{z^{2}}+\epsilon\right] d z\right| \leq K \int_{\pi / 2}^{3 \pi / 2} e^{T \lambda(L)^{-1} \cos \phi} d \phi .
$$




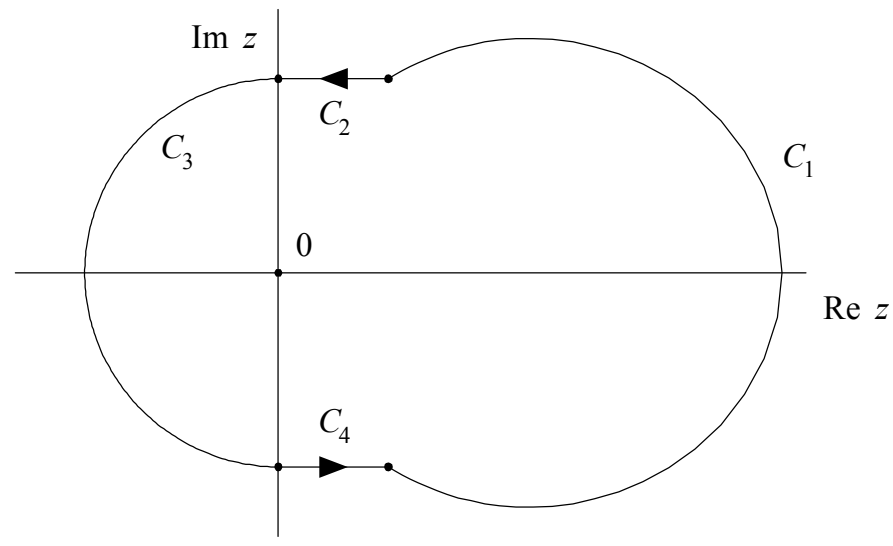

Figure 3.3: The closed contour $C$ in the $z$-plane, traversed clockwise. The origin $z=0$ is an isolated essential singularity of the integrand. No other singularities are enclosed.

The change of variable $\phi=\theta+\pi / 2$ and the observation that $\sin \theta \geq 2 \theta / \pi$ for $0 \leq \theta \leq \pi / 2$ gives

$$
\begin{aligned}
\int_{\pi / 2}^{3 \pi / 2} e^{T \lambda(L)^{-1} \cos \phi} d \phi & =\int_{0}^{\pi} e^{-T \lambda(L)^{-1} \sin \theta} d \theta=2 \int_{0}^{\pi / 2} e^{-T \lambda(L)^{-1} \sin \theta} d \theta \\
& \leq 2 \int_{0}^{\pi / 2} e^{-T \lambda(L)^{-1} 2 \theta / \pi} d \theta \leq 2 \int_{0}^{\infty} e^{-T \lambda(L)^{-1} 2 \theta / \pi} d \theta \\
& =\frac{\pi \lambda(L)}{T} \rightarrow 0 \quad \text { as } L \rightarrow \infty,
\end{aligned}
$$

and we have proved that the integral over $C_{3}$ vanishes in the limit $L \rightarrow \infty$.

The estimation of the integrals over $C_{2}$ and $C_{4}$ is similar, and we write out only the estimate for $C_{2}$. On the contour $C_{2}$, we write $z=\chi(L) \xi+i \lambda(L)$, where $0 \leq \xi \leq 1$. Since

$$
\operatorname{Re}\left\{z^{-1} T\right\}=\operatorname{Re}\left\{\frac{T}{\chi(L) \xi+i \lambda(L)}\right\}=\frac{T \chi(L) \xi}{(\chi(L) \xi)^{2}+\lambda(L)^{2}} \leq \frac{T \chi(L)}{\lambda(L)^{2}}
$$

and $\chi(L) / \lambda(L)^{2} \rightarrow 1+\kappa+\epsilon$ as $L \rightarrow \infty$ we see that

$$
\left|\exp \left(z^{-1} T\right)\right|=\exp \left(\operatorname{Re}\left\{z^{-1} T\right\}\right)=O(1) .
$$

The remaining factors in the integrand are easily bounded and we readily show that the integral vanishes in the limit $L \rightarrow \infty$.

We have now proved that

$$
\begin{aligned}
& \lim _{L \rightarrow \infty} \frac{1}{2 \pi i} \int_{C_{1}} e^{\left(z^{-1}+\epsilon z-1-\epsilon\right) T} z e^{-\alpha u+\alpha u z}\left[-\frac{1}{z^{2}}+\epsilon\right] d z \\
& =e^{-\alpha u-(1+\epsilon) T}\left\{I_{0}(\sqrt{4 T(\epsilon T+\alpha u)})-\frac{T}{T+\alpha u} I_{2}(\sqrt{4 T(\epsilon T+\alpha u)})\right\} .
\end{aligned}
$$


If we make the change of variable the left-hand side becomes

$$
\lim _{L \rightarrow \infty} \frac{1}{2 \pi i} \int_{\kappa-i L}^{\kappa+i L} e^{s T} \widehat{f}(s) d s=f(T)
$$

and so we have established the validity of the contour integral approach introduced for Erlang parameter $n=1$ by Garcia (2002), leading to the final answer

$$
\begin{aligned}
\frac{\partial}{\partial t} \psi(u, t)= & \beta e^{-\alpha u-(\beta+c \alpha) t} \times \\
& \left\{I_{0}(\sqrt{4 \alpha \beta c t(t+u / c)})-\frac{t}{t+u / c} I_{2}(\sqrt{4 \alpha \beta c t(t+u / c)})\right\} .
\end{aligned}
$$

Using the asymptotic expansion (Abramowitz and Stegun 1965, equation $(9.7 .1))$

$$
I_{\nu}(Z)=\frac{e^{Z}}{\sqrt{2 \pi Z}}\left\{1-\frac{4 \nu^{2}-1}{8 Z}+O\left(Z^{-2}\right)\right\}
$$

for $Z \rightarrow \infty$, we are able to verify that this form of the answer is consistent with the asymptotic estimate (3.7). As modified Bessel functions are easily extracted from tables or computed with standard software, equation (3.9) represents a convenient practical solution of the problem for $n=1$, and is preferable to the Drekic-Willmot series. We remark that producing the asymptotic form directly from the Drekic-Willmot series appears very difficult.

The equivalence of equations (3.8) and (3.9) establishes indirectly the identity

$I_{0}(\sqrt{z(z+2 \zeta)})-\frac{z I_{2}(\sqrt{z(z+2 \zeta)})}{z+2 \zeta}=\frac{1}{\pi} \int_{-\pi}^{\pi} e^{(z+\zeta) \cos \phi} \sin (\zeta \sin \phi+\phi) \sin \phi d \phi$.

\section{The case of general $n$ : $z$-plane analysis}

It is tempting to assert the unambiguous global existence of $Z(s)$, but this is not a trivial point. A function $F(z)$ is called univalent in a region of the $z$ plane if $F\left(z_{1}\right)=F\left(z_{2}\right)$ implies that $z_{1}=z_{2}$. From the theory of univalent functions (see Henrici 1986, pp. 571-584, especially Theorem 19.1g) it may be proved that $P_{s}(z)$ is not univalent in $|z|<1$ if $n \epsilon^{2}>1$. However, as the explicit determination of $Z(s)$ is impossible for $n \geq 5$, we cannot resolve this question rigorously for arbitrary $n$ by elementary means.

However, apart from this caveat, the generalisation of the evaluation of $f(T)$ via an integral in the $z$-plane to $n>1$ is straightforward, with a contour similar to that in Figure 3.3 used. Although we cannot exhibit $Z(s)$ explicitly, it is easy to construct the large- $|s|$ asymptotic solution of the equation

$$
Z(s)\left[1+s+\epsilon\left(1-Z(s)^{n}\right)\right]=1
$$

directly and verify that for all integer $n \geq 1$, as $L \rightarrow \infty$

$$
Z(\kappa+i L)=-\frac{i}{L}+\frac{1+\kappa+\epsilon}{L^{2}}+O\left(L^{-3}\right) .
$$


The argument used to bound the contribution from the semicircular arc $C_{3}$ on the left of the imaginary axis extends immediately. The analysis of the contributions from $C_{2}$ and $C_{4}$ is also straightforward, with the result that $\operatorname{Re}\{Z(\kappa+i L)\} / \operatorname{Im}\{Z(\kappa+i L)\}^{2}$ is bounded as $L \rightarrow \infty$ playing a crucial role. We find that

$$
\begin{gathered}
f(T)=\underset{z=0}{-\operatorname{Residue}}\left\{e^{\left(z^{-1}+\epsilon z^{n}-1-\epsilon\right) T} z^{n} e^{-\alpha u+\alpha u z^{n}}\left[-\frac{1}{z^{2}}+\epsilon n z^{n-1}\right]\right\} \\
=e^{-\alpha u-(1+\epsilon) T} \text { Residue }\left\{e^{z^{-1} T} z^{n} e^{(\epsilon T+\alpha u) z^{n}}\left[\frac{1}{z^{2}}-\epsilon n z^{n-1}\right]\right\} \\
z=0 \\
=e^{-\alpha u-(1+\epsilon) T}\left\{\sum_{m=0}^{\infty} \frac{(\epsilon T+\alpha u)^{m} T^{n(m+1)-1}}{[n(m+1)-1] ! m !}\right. \\
\left.-\epsilon n \sum_{m=0}^{\infty} \frac{(\epsilon T+\alpha u)^{m} T^{n(m+2)}}{[n(m+2)] ! m !}\right\} .
\end{gathered}
$$

This can be rewritten as

$$
\begin{aligned}
\frac{\partial}{\partial t} \psi(u, t)=\beta e^{-\alpha u-(\beta+\alpha c) t} & \left\{(\beta t)^{n-1} \sum_{m=0}^{\infty} \frac{(\alpha c t+\alpha u)^{m}(\beta t)^{n m}}{[n(m+1)-1] ! m !}\right. \\
& \left.-\frac{\alpha c n}{\beta}(\beta t)^{2 n} \sum_{m=0}^{\infty} \frac{(\alpha c t+\alpha u)^{m}(\beta t)^{n m}}{[n(m+2)] ! m !}\right\} .
\end{aligned}
$$

The numerical evaluation of these series is straightforward. We can rewrite the answer we have obtained in terms of the generalised hypergeometric function

$$
{ }_{0} F_{q}\left(C_{1}, C_{2}, \ldots C_{q} ; Z\right)=\sum_{m=0}^{\infty} \frac{\Gamma\left(C_{1}\right) \Gamma\left(C_{2}\right) \cdots \Gamma\left(C_{q}\right)}{\Gamma\left(C_{1}+m\right) \Gamma\left(C_{2}+m\right) \cdots \Gamma\left(C_{q}+m\right)} \frac{Z^{m}}{m !} .
$$

To do this, we use the multiplication formula of Gauss (Whittaker and Watson 1927, p. 240)

$$
(n Z-1) !=\Gamma(n Z)=(2 \pi)^{(1-n) / 2} n^{n Z-1 / 2} \prod_{k=0}^{n-1} \Gamma(Z+k / n)
$$

with $Z=m+1$ and with $Z=m+2+1 / n$ to establish the identities

$$
\begin{aligned}
\frac{(n-1) !}{[n(m+1)-1] !} & =\frac{1}{n^{n m}} \prod_{k=0}^{n-1} \frac{\Gamma(1+k / n)}{\Gamma(m+1+k / n)}, \\
\frac{(2 n) !}{[n(m+2)] !} & =\frac{1}{n^{n m}} \prod_{k=0}^{n-1} \frac{\Gamma(2+(k+1) / n)}{\Gamma(m+2+(k+1) / n)},
\end{aligned}
$$

from which we deduce that

$$
\begin{aligned}
\frac{\partial}{\partial t} \psi(u, t)= & \beta e^{-\alpha u-(\beta+\alpha c) t} \times \\
& \left\{\frac{(\beta t)^{n-1}}{(n-1) !}{ }^{0} F_{n}\left(1,1+\frac{1}{n}, \cdots, 1+\frac{n-1}{n} ; \frac{\alpha(c t+u)(\beta t)^{n}}{n^{n}}\right)\right. \\
& \left.-\frac{\alpha c n(\beta t)^{2 n}}{(2 n) ! \beta}{ }_{0} F_{n}\left(2+\frac{1}{n}, 2+\frac{2}{n}, \cdots, 2+\frac{n}{n} ; \frac{\alpha(c t+u)(\beta t)^{n}}{n^{n}}\right)\right\} .
\end{aligned}
$$




\begin{tabular}{ccccc}
\hline$t$ & $n=1$ & $n=2$ & $n=3$ & $n=4$ \\
\hline \hline 1 & 0.0003 & 0.0001 & 0.0000 & 0.0000 \\
2 & 0.0013 & 0.0004 & 0.0002 & 0.0001 \\
3 & 0.0032 & 0.0010 & 0.0006 & 0.0004 \\
4 & 0.0059 & 0.0019 & 0.0012 & 0.0009 \\
5 & 0.0092 & 0.0033 & 0.0020 & 0.0016 \\
10 & 0.0319 & 0.0145 & 0.0100 & 0.0081 \\
20 & 0.0822 & 0.0457 & 0.0346 & 0.0294 \\
30 & 0.1242 & 0.0756 & 0.0597 & 0.0519 \\
40 & 0.1573 & 0.1008 & 0.0814 & 0.0718 \\
50 & 0.1837 & 0.1215 & 0.0997 & 0.0887 \\
100 & 0.2605 & 0.1842 & 0.1557 & 0.1410 \\
200 & 0.3178 & 0.2311 & 0.1976 & 0.1801 \\
300 & 0.3398 & 0.2482 & 0.2125 & 0.1937 \\
400 & 0.3505 & 0.2559 & 0.2190 & 0.1996 \\
500 & 0.3564 & 0.2599 & 0.2223 & 0.2024 \\
1,000 & 0.3649 & 0.2647 & 0.2259 & 0.2055 \\
$\infty$ & 0.3663 & 0.2652 & 0.2262 & 0.2058 \\
\hline
\end{tabular}

Table 4.1: Values of $\psi(10, t)$ when $\varpi=10 \%$.

To use standard software to compute the ${ }_{0} F_{q}$ functions when they are not directly available, we note that for all $M$ except $0,-1,-2, \ldots$

$$
{ }_{0} F_{q}\left(C_{1}, C_{2}, \ldots C_{q} ; Z\right)={ }_{1} F_{q+1}\left(M ; M, C_{1}, C_{2}, \ldots C_{q} ; Z\right)
$$

\section{Numerical Examples}

In this section we give some numerical values, calculated by numerical integration of the density of $T_{c}$ using Mathematica.

Table 4.1 shows values of $\psi(10, t)$ to four decimal places for a range of values of $t$ when the premium loading factor $\varpi$ is 0.1 , the Erlang parameter is $n=$ $1,2,3,4$ and $\beta=n$, so that the mean inter-arrival time is 1 in each case. We observe from Table 4.1 that as $n$ increases, for given values of $u$ and $t, \psi(u, t)$ decreases. This is not surprising as an increase in $n$ causes a decrease in the variance of $W_{1}$. Table 4.2 is as Table 4.1 except that we have set $\varpi=0.25$. We observe the same features in Table 4.2 as in Table 4.1. Additionally, we note that for a given value of $n$, convergence to the ultimate ruin probability is faster than in Table 4.1.

Figure 4.1 shows a plot of the density of $T_{c}$ when $u=40$ and $\varpi=0.1$ for $n=1,2,3,4$ and $\beta=n$. For this choice of parameters, the ultimate ruin probability decreases from 0.0240 when $n=1$ to 0.0028 when $n=4$. Figure 4.2 shows a similar plot, but with $u=20$ and $\varpi=0.25$, so that the ultimate ruin probability decreases from 0.0147 when $n=1$ to 0.0015 when $n=4$. In these figures, the case $n=1$ corresponds to Figures 1 and 2 in Dickson and Waters (2002). We note the features are similar in Figures 4.1 and 4.2, with an increase in skewness as $n$ increases. It is clear from Figures 4.1 and 4.2 that if ruin occurs, it is likely to occur sooner when $\varpi=0.25$ than when $\varpi=0.1$. 


\begin{tabular}{ccccc}
\hline$t$ & $n=1$ & $n=2$ & $n=3$ & $n=4$ \\
\hline \hline 1 & 0.0003 & 0.0001 & 0.0000 & 0.0000 \\
2 & 0.0012 & 0.0003 & 0.0002 & 0.0001 \\
3 & 0.0026 & 0.0007 & 0.0004 & 0.0003 \\
4 & 0.0046 & 0.0014 & 0.0008 & 0.0006 \\
5 & 0.0069 & 0.0023 & 0.0014 & 0.0010 \\
10 & 0.0209 & 0.0084 & 0.0055 & 0.0043 \\
20 & 0.0464 & 0.0217 & 0.0151 & 0.0122 \\
30 & 0.0640 & 0.0315 & 0.0225 & 0.0184 \\
40 & 0.0760 & 0.0383 & 0.0276 & 0.0228 \\
50 & 0.0842 & 0.0430 & 0.0311 & 0.0257 \\
100 & 0.1016 & 0.0522 & 0.0378 & 0.0312 \\
200 & 0.1075 & 0.0547 & 0.0394 & 0.0324 \\
300 & 0.1081 & 0.0549 & 0.0395 & 0.0325 \\
400 & 0.1082 & 0.0549 & 0.0395 & 0.0325 \\
500 & 0.1083 & 0.0549 & 0.0395 & 0.0325 \\
1,000 & 0.1083 & 0.0549 & 0.0395 & 0.0325 \\
$\infty$ & 0.1083 & 0.0549 & 0.0395 & 0.0325 \\
\hline
\end{tabular}

Table 4.2: Values of $\psi(10, t)$ when $\varpi=25 \%$.

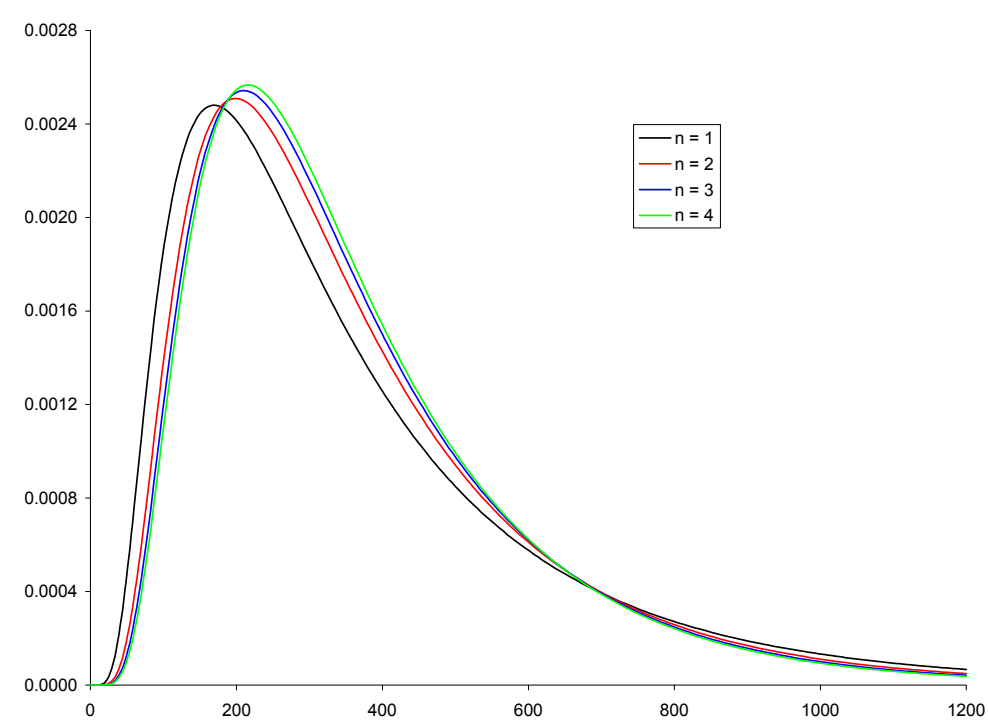

Figure 4.1: Densities of $T_{c}$ when $u=40$ and $\varpi=0.1$. 


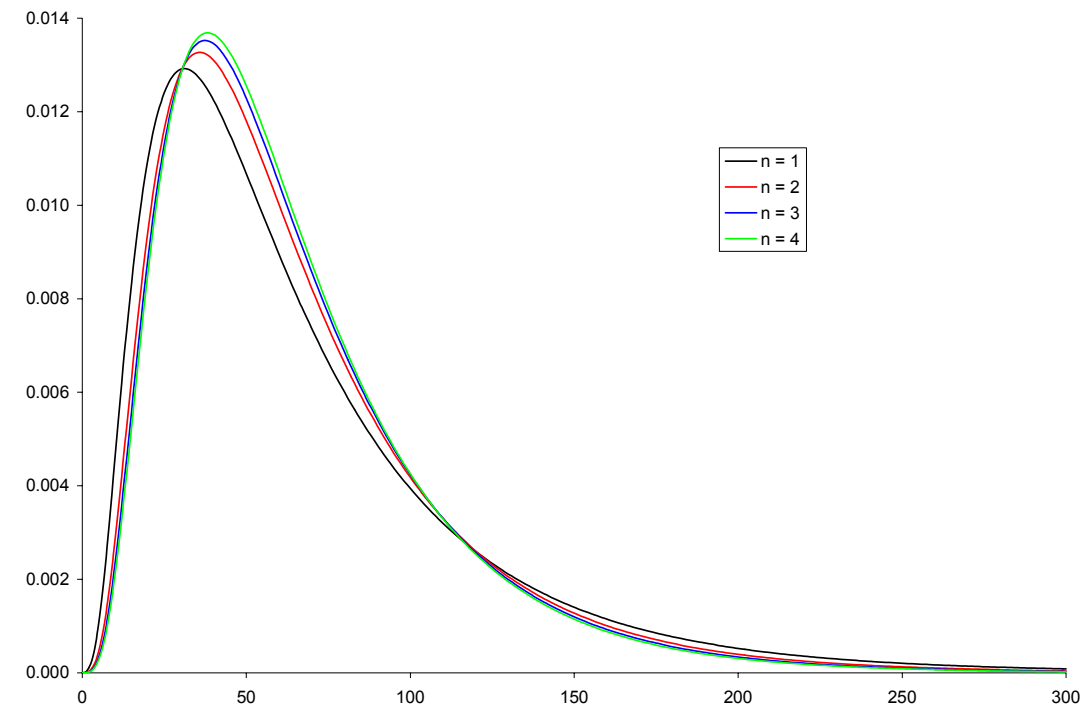

Figure 4.2: Densities of $T_{c}$ when $u=20$ and $\varpi=0.25$.

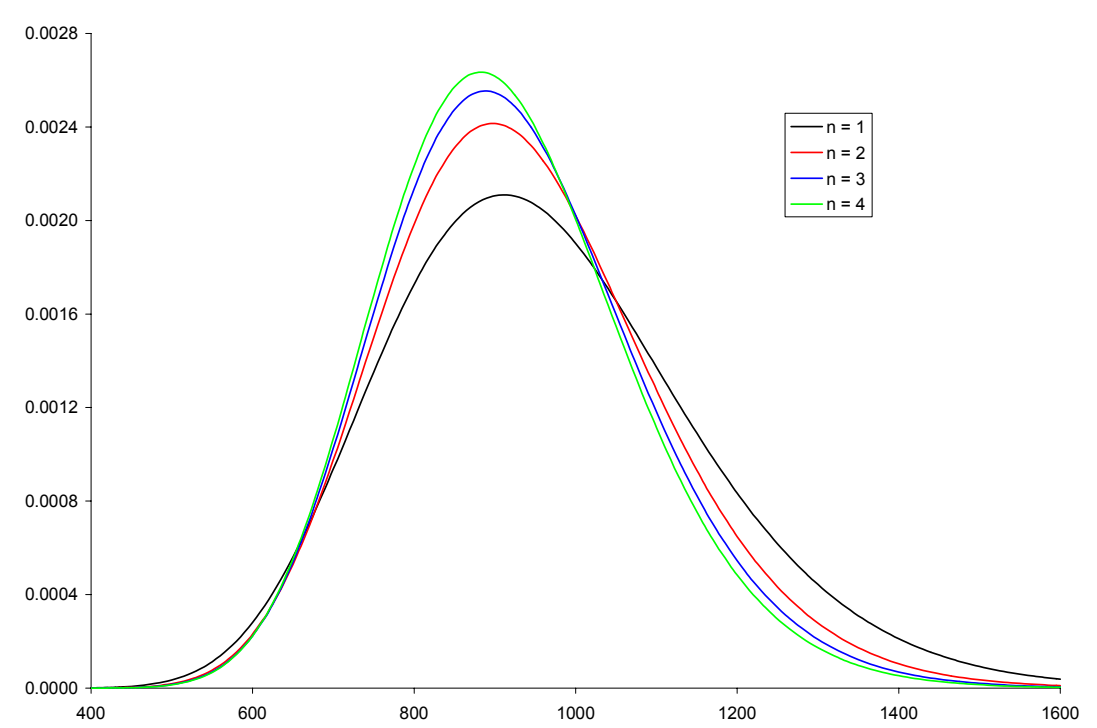

Figure 4.3: Densities of $T_{c}$ when $u=300$ and $\varpi=0.25$. 
Figure 4.3 shows a plot of the density of $T_{c}$ when $u=300$ and $\varpi=0.25$ for $n=1,2,3,4$ and $\beta=n$. For this choice of parameter values the largest ultimate ruin probability is $7 \times 10^{-27}$, a value that is clearly of no practical interest. However, we note that for each value of $n$, the density of $T_{c}$ is no longer skewed as in Figures 4.1 and 4.2, but appears to be normal, in line with the well known result of Segerdahl (1955) in the context of the classical risk model.

\section{References}

[1] Abramowitz, M. and Stegun, I.A. (1965) Handbook of Mathematical Functions. Dover, New York.

[2] Dickson, D.C.M. and Hipp, C. (2001) On the time to ruin for Erlang(2) risk processes. Insurance: Mathematics and Economics 29, 333-344.

[3] Dickson, D.C.M. and Waters, H.R. (2002) The distribution of the time to ruin in the classical risk model. ASTIN Bulletin 32, 299-313.

[4] Drekic, S. and Willmot, G.E. (2003) On the density and moments of the time of ruin with exponential claims. ASTIN Bulletin 33, 11-21.

[5] Garcia, J.M.A. (2002) Explicit solutions for survival probabilities in a finite time horizon. Presented to the 6th International IME Congress, Lisbon. http://pascal.iseg.utl.pt/ cemapre/ime2002/index.html (No. 49).

[6] Gerber, H.U. and Shiu, E.S.W. (1998) On the time value of ruin. North American Actuarial Journal 2, 48-78.

[7] Grandell, J. (1991) Aspects of Risk Theory. Springer-Verlag, New York.

[8] Henrici, P. (1977) Applied and Computational Complex Analysis, Volume 2. John Wiley \& Sons, New York.

[9] Henrici, P. (1986) Applied and Computational Complex Analysis, Volume 3. John Wiley \& Sons, New York.

[10] Marsden, J.E. and Hoffman, M.J. (1999) Basic Complex Analysis, 3rd edition. W.H. Freeman, New York.

[11] Olver, F.W.J. (1974) Asymptotics and Special Functions. Academic Press, New York.

[12] Seal, H.L. (1978) Survival probabilities: the goal of risk theory. John Wiley \& Sons, New York.

[13] Segerdahl, C.-O. (1955) When does ruin occur in the collective theory of risk? Skandinavisk Aktuarietidskrift XXXVIII, 22-36.

[14] Sneddon, I.N. (1972) The Use of Integral Transforms. McGraw-Hill, New York.

[15] Whittaker, E.T. and Watson, G.N. (1927) A Course of Modern Analysis, 2nd edition. Cambridge University Press. 


\author{
David C M Dickson \\ Centre for Actuarial Studies \\ Department of Economics \\ University of Melbourne \\ Victoria 3010 \\ Australia \\ email: dcmd@unimelb.edu.au \\ Barry D Hughes \\ Department of Mathematics and Statistics \\ University of Melbourne \\ Victoria 3010 \\ Australia \\ email: B.Hughes@ms.unimelb.edu.au \\ Zhang Lianzeng \\ Department of Risk Management and Insurance \\ Nankai University \\ Tianjin 300071 \\ PR China \\ email: lianzeng@public.tpt.tj.cn
}




\section{University Library}

\section{- M M N E R VA A gateway to Melbourne's research publications}

Minerva Access is the Institutional Repository of The University of Melbourne

Author/s:

Dickson, David C. M.;Hughes, Barry D.;Lianzeng, Zhang

Title:

The density of the time to ruin for a Sparre Andersen process with Erlang arrivals and exponential claims

Date:

2003-10

Citation:

Dickson, David C. M. and Hughes, Barry D. and Lianzeng, Zhang (2003) The density of the time to ruin for a Sparre Andersen process with Erlang arrivals and exponential claims.

Persistent Link:

http://hdl.handle.net/11343/34348 